ISSN electrónico: 2172-9077

DOI: https://doi.org/10.14201/fjc-v22-22664

\title{
LA COMUNICACIÓN ONLINE DE LA RSC EN MATERIA MEDIOAMBIENTAL. EL CASO DE EMPRESAS DE MODA RÁPIDA Y MODA LENTA
}

\section{Online CSR communication on environmental responsibility. The case of fast fashion and slow fashion companies}

\author{
Laura GARCÍA-HUGUET \\ Personal Investigador en Formación, Universitat Jaume I \\ E-mail: lauragarciahuguet@hotmail.com \\ (iD) https://orcid.org/0000-0003-2601-6660
}

Fecha de recepción del artículo: 10/03/2020

Fecha de aceptación definitiva: 30/03/2020

\begin{abstract}
RESUMEN
La preocupación de la ciudadanía por el impacto medioambiental de sus acciones les ha llevado a replantear sus hábitos de consumo hacia unos más sostenibles con su entorno. Las empresas, como entes sociales y tanto por este motivo como por su impacto medioambiental, social y económico, han de replantear su estrategia de negocio hacia una más sostenible y comprometida con estas tres esferas en un paradigma empresarial en la que la correcta gestión de los intangibles, como la Responsabilidad Social Corporativa, puede inferir en una buena reputación. Sobre todo, en el caso de la moda rápida, que se convierte en una de las más contaminantes del mundo. El presente trabajo pretende analizar las comunicaciones sobre RSC que estas empresas comparten en Facebook y si existe una hegemonía o diferencia entre las comunicaciones de este tipo que realizan las organizaciones de moda lenta, con un modelo de producción y confección totalmente sostenible. De esta investigación se concluye que, a pesar de realizar acciones sostenibles como informan en sus páginas corporativas, las empresas de moda rápida no las comunican en su perfil de Facebook, a diferencia de las de moda lenta que las comunican de forma constante.
\end{abstract}

Palabras clave: Responsabilidad Social Corporativa; Valores Intangibles; Comunicación Corporativa; Comunicación de Moda; Moda; Redes Sociales.

\begin{abstract}
Citizens' concern about the environmental impact of their actions has led them to rethink their consumption habits towards more sustainable ones regarding their environment. Companies, as social entities and both for this reason and for their environmental, social and economic impact, have to rethink their business strategy towards a more sustainable one which is more committed to these three spheres in a business paradigm in which the correct management of the intangible values, such as Corporate Social Responsibility, can result in a good reputation. Above all, in the case of fast fashion, which is one of the most polluting ones in the world. This paper intends to analyse the communications about CSR
\end{abstract}




\begin{abstract}
that these companies share on Facebook and whether there is a hegemony or difference compared with communications of this type carried out by slow fashion organizations, with a totally sustainable production and manufacturing model. This research concludes that, despite performing sustainable actions as reported in their corporate websites, fast fashion companies do not communicate them on their Facebook profile, unlike slow fashion companies, which com-municate them constantly.
\end{abstract}

Keywords: Social Corporate Responsibility; Intangible values; Corporate Communication; Fashion Communication; Fashion; Social Media.

\title{
1. Introducción
}

La emergencia climática es, en la actualidad, uno de los temas que más preocupan a la agenda pública. Las amenazas contra el medio ambiente y el cambio climático se sitúan en décima y duodécima posición, respectivamente, en los temas que más preocupan a la población mundial, según el estudio 'What Worries the World', de Ipsos Global Advisor (2019, p. 7). Además, los ciudadanos son conscientes de que el calentamiento global es un proceso inducido por la actividad humana, y no como un proceso natural de la Tierra (Fundación BBVA, 2019, p. 80).

Movimientos como el Fridays for Future, iniciado a raíz de las protestas de la joven activista Greta Thunberg, reclaman la protección de un ecosistema en grave peligro mediante manifestaciones alrededor de todo el mundo antes de que la situación sea irreversible. Los programas informativos incluyen noticias sobre este tema en su parrilla diaria, e incluso en ocasiones dedican secciones especiales a ello, como el caso de La Sexta Noticias durante la celebración de la Cumbre del Clima en Madrid el pasado mes de diciembre de 2019.

Por otro lado, los influencers comparten en sus perfiles de redes sociales ${ }^{1}$ sus hábitos de vida sostenibles, llegando estos mensajes a millones de usuarios. Una audiencia que «se ha vuelto hoy en día más selectiva, exigente y formada-informada si cabe, debido a la multicanalidad y la multiplicidad de mensajes y fuentes» (Castelló, 2010a, p. 101), más comprometida con su entorno (OCU, 2019, p. 6) y consciente de que las empresas, además de las administraciones y la propia sociedad, tienen una gran responsabilidad frente a los problemas medioambientales (Tetra Pak, 2019, p. 7).

Las organizaciones, como parte de la sociedad, deben fomentar y aplicar, de forma voluntaria y no solamente por imperativo legal, medidas a favor de su entorno (económico, social y medioambiental). Ese compromiso para con la sociedad se designa como Responsabilidad Social Corporativa ${ }^{2}$, y se configura como uno de los intangibles que generan valor a una empresa, junto a la Marca, la Gestión del Conocimiento o la Reputación Corporativa (Villafañe, 2005, p. 104), entre otros.

Y, ahora más que nunca, la gestión de la RSC se convierte en un elemento diferenciador para las organizaciones; no sólo por las demandas del nuevo consumidor, sino también para distinguirse de sus competidores «en un entorno cada vez más saturado y globalizado" (Benavides y Villagra, 2005, p. 154) y recuperar la confianza perdida de la sociedad. Por supuesto, no es sólo importante realizar estas acciones en pos del beneficio social, sino que su implantación se ha de comunicar a los grupos de interés

1. En adelante, RRSS.

2. En adelante, RSC. 
(stakeholders), pues de nada sirve realizar medidas que pueden ser de su interés sin comunicárselo.

En concreto, el sector de la moda está tomando riendas en el camino hacia la sostenibilidad. Siendo el segundo sector más contaminante del mundo, como afirmó la ONU en su Conferencia sobre Comercio y Desarrollo, y en contraposición al modelo de producción de las empresas de moda rápida, observamos cómo, cada vez, se crean más empresas de moda lenta (aunque algunas ya llevan en el sector desde hace dos décadas), y que se diferencian de las primeras por un ritmo de producción más tardío y en la confección de sus prendas, producidas de forma sostenible y fabricadas con materiales biodegradables.

Por su parte, las grandes empresas de moda rápida están implantando medidas para reducir su huella ambiental: colecciones sostenibles diferenciadas por etiquetas del resto de la producción (que sigue siendo no sostenible), medidas relacionadas con la reducción de emisiones de $\mathrm{CO} 2$, la reducción del gasto de agua en el proceso de producción o el uso de materias primas orgánicas, todas ellas recogidas en informes de sostenibilidad elaborados de forma anual.

Ahora, nos preguntamos: ¿en qué medida las empresas de moda rápida comunican su responsabilidad medioambiental, a sus stakeholders, en el entorno online? ¿Hay alguna diferencia o similitud entre las comunicaciones de este tipo llevadas a cabo por parte de empresas de moda rápida y moda lenta? Responder a estas preguntas es lo que se pretende con el desarrollo de la presente investigación. La importancia de la misma radica en el hecho del gran impacto ambiental del sector de la moda, sumado al carácter de un nuevo consumidor, que se informa sobre todo lo que le preocupa. Y, siendo el estado del medio ambiente una de las mayores inquietudes de la sociedad actual, analizar cómo se informa a los usuarios sobre políticas sostenibles por parte de ambos tipos de empresa es algo que, hasta el momento, no se había realizado en ningún estudio y que sitúa al tema analizado en pleno centro de la actualidad. Por eso, además, solo tenemos en cuenta la esfera medioambiental en el análisis de dichas comunicaciones, aunque entendemos que las esferas social y económica no deben ser olvidadas a la hora de implantar acciones de RSC.

Porque, es cierto que existen investigaciones que, o bien analizan la presencia en la web de empresas de moda españolas (Vaño, Calduch-Losa y Orduña-Malea, 2016), o el uso de RRSS y su reputación digital, en el caso de Zara y H\&M (Fondevila, Del Olmo y Bravo, 2012), o las estrategias comunicativas llevadas a cabo en Facebook por parte de Zara y Mango (Cristófol, Segarra-Saavedra y Cristófol-Rodríguez, 2019), pero encontramos un vacío en lo referido al análisis de la comunicación sobre RSC en empresas de moda, ya sea moda rápida o moda lenta; un vacío que intenta llenarse mediante este estudio, de ahí su relevancia.

\section{La RSC: un intangible imprescindible}

La Comisión Europea define la RSC como «la integración voluntaria, por parte de las empresas, de objetivos sociales y medioambientales en sus operaciones comerciales y en sus relaciones con el resto de actores implicados" (2002) para "contribuir al logro de una sociedad mejor y un medio ambiente más limpio» (Comisión Europea, 2001, p. 8).

Aunque ambas definiciones sólo contemplan dos esferas de la RSC, diversos autores señalan que sus áreas de actuación se resumen en tres, y serían aquellas en 
las que el ejercicio de la empresa genera impacto (Velasco, 2006, p. 22): aparte de las anteriormente citadas, también se contempla una dimensión económica (Kliksberg, 2012; Fernández y Muñoz Torres, 2008 o Iborra-Juan y Peris-Suay (2010), entre otros) y que, en su conjunto, forman la triple cuenta de resultados, cuya eficaz gestión proporciona «beneficios adicionales [...] relacionados con la credibilidad, la imagen y la reputación [...] y son los que están marcando la diferencia entre organizaciones» (Túñez y Valarezo, 2012, p. 63).

Dicha triple cuenta de resultados fue definida por John Elkington en su libro 'Cannibals with Forks' (1997), en el cual su autor argumenta que, si una empresa quiere ser sostenible, «tiene que garantizar un triple objetivo: ser económicamente viable, ser socialmente beneficiosa y ser ambientalmente responsable», como añaden Andreu y Fernández (2011, pp. 13-14). Ahora, la consideración de esta tridimensionalidad en la gestión empresarial, ha provocado que las memorias del negocio pasen de comunicar, a sus stakeholders, únicamente aspectos económicos para informarles también sobre aspectos ambientales y sociales (Velasco, 2006, p. 24).

A su vez, no debe confundirse la RSC con diversas actividades de índole social que también puede llevar a cabo una organización, tal y como explica Barrio (2019): la acción social, que tan sólo «supone una pequeña parte de la RSC, ya que implica la realización voluntaria de acciones en materia social con el fin de dar respuesta a los intereses por parte de la sociedad»; la filantropía, consistente en «donaciones [...] a beneficiarios que no tienen porqué estar relacionados con la actividad de la empresa"; el marketing con causa, utilizado "con el fin de ayudar a la venta de un producto o de un servicio, utilizando para ello una buena causa" o la ética, que se ocupa de "la reflexión sobre cuáles deben ser los criterios de actuación y valores que guíen el desarrollo de la actividad de la organización", mientras que la RSC "se centra en las acciones concretas y cómo llevarlas a cabo» (pp. 57-67).

La RSC se diferencia, por tanto, de las mismas porque éstas «no implican un cambio organizacional, ni mucho menos constituyen un modelo de gestión sostenida en virtud de alcanzar el beneficio particular sin descuidar el colectivo» (Túñez y Valarezo, 2012, p. 63), sino que la RSC ha de ser vista "como una herramienta de gestión, como una medida de prudencia y como una exigencia de justicia” (Cortina, 2005, p. 3) y "como una palanca de creación de valor en el core business de la empresa» (Andreu y Fernández, 2011, p. 8).

En la literatura, los orígenes de la RSC se remontan a los años 50, siendo Bowen (1953) el primero en hacer referencia a la misma como un cometido de los hombres de negocios. Por su parte, también hubo detractores como Milton Friedman quien, en 1970, afirmaba, como bien explica Cortina (2005), que «la responsabilidad social consiste en aumentar el beneficio para el accionista, porque la empresa es un instrumento del accionista, que es su propietario» (p. 2), una doctrina que ha evolucionado, pues «la empresa prudente intuye que si tiene en cuenta los intereses de los afectados en el diseño de las estrategias de la empresa, también aumentará el beneficio del accionista” (ibíd., p. 3). Además, Fernández (2005) destaca que, en el entorno capitalista actual, la tesis de Friedman «no va a resultar suficiente [...] porque los consumidores -e incluso los inversores, tanto privados, cuanto institucionales- no lo van a permitir» (p. 27).

La RSC alcanza tanta importancia en las organizaciones, por causa principal, debido a la preocupación de la ciudadanía por su entorno, por lo que demanda a las organizaciones una conducta y unas prácticas éticas, así como una gestión de sus 
actividades más responsable (Castelló, 2010b, p. 26). Dicha demanda viene dada a raíz de los diversos escándalos relacionados con la corrupción o violaciones de derechos humanos en diversas empresas de índole internacional en los años 90 (Jáuregui, 2012, p. 27), pero también numerosos escándalos más recientes, así como los diversos métodos desarrollados para conseguir que las empresas sean rentables (Tuñez y Valarezo, 2012, p. 62), que, conjuntamente, han provocado una pérdida de confianza en las empresas.

Entre otros motivos por los que surge la RSC, como señalan diversos investigadores, se sitúan: en primer lugar, la globalización y los cambios, tanto sociales como económicos, producidos por la misma (Gómez y Martínez, 2015, p. 5); seguidamente, la evolución de las TIC que, además, ha propiciado la aparición de un nuevo consumidor más informado y exigente y, por último, la redefinición de las empresas a partir de la teoría de los stakeholders (Barrio, 2019, pp. 15-25), formulada por Edward Freeman $^{3}$, en la cual los mismos «ejercen el derecho de voz que establece medidas de control y de responsabilidad (accountability)» (González, 2007, p. 218), siendo considerados "como ciudadanos que pueden y deben exigir los derechos de ciudadanía económica, social y política» (ibíd., p. 207).

Pero fue la creación del Pacto Mundial de las Naciones Unidas, en el Foro Económico de Davos celebrado el 31 de enero de 1999, la que «supuso un antes y un después en materia de RSC» (Andreu y Fernández, 2011, p. 6). En el mismo, el entonces secretario general de las Naciones Unidas, Kofi Annan, propuso a las empresas el seguimiento de unos principios en materia de derechos humanos, trabajo, anticorrupción y medio ambiente, con el objetivo de «ayudar a fortalecer los cimientos sociales en los cuales debe estar arraigado cualquier mercado, incluyendo el mercado mundial, si la idea es sobrevivir y prosperar» y a favor de los «valores universales y de la práctica de actividades empresariales con alto sentido de responsabilidad" (Naciones Unidas).

A raíz del lanzamiento del Pacto Mundial, se crearon diversos instrumentos relacionados con la sostenibilidad de los negocios, como el Dow Jones Sustainability Index (1999), el Global Reporting Iniciative (GRI) (2000), las Directrices de la OCDE para Empresas Multinacionales (2000), la renovación de la Declaración tripartita de la OIT en materia de RSC (creada en 1977, renovada en el año 2000), el Libro Verde de la Comisión Europea (2001), entre muchos otros, así como diversas certificaciones, como ISO14001 o ISO50001, en materia medioambiental, o SA8000 e ISO26000, relacionadas con la responsabilidad social de las organizaciones.

En 2015, la Asamblea General de Naciones Unidas aprobó la Agenda 2030 sobre el Desarrollo Sostenible, un plan de acción «a favor de las personas, el planeta y la prosperidad“ (Naciones Unidas, 2015), compuesta por 17 Objetivos de Desarrollo Sostenible (ODS) que abarcan las tres esferas de la RSC y se dirigen al conjunto de la sociedad: administraciones, ciudadanos y el sector privado. De hecho, se ha elaborado un documento-guía para que las empresas puedan realizar acciones para cumplimentar dichos objetivos, ejemplificando con buenas prácticas.

Es tal la relevancia actual de la RSC en el actual paradigma empresarial que diversos medidores reputacionales incluyen variables de medición que contemplan la sostenibilidad a la hora de valorar la reputación de las empresas. En Merco (2019), las ONG y los periodistas económicos son los grupos de expertos que evalúan la dimensión 'Ética y Responsabilidad Corporativa', con un peso final en la ponderación del

3. Freeman, R.E. (1984). Strategic management. A Stakeholder Approach. London: Pitman 
15,3\%, y RepTrak (2019) resume en tres las dimensiones (de un total de siete) relacionadas con la RSC: Ciudadanía, Integridad y Trabajo, y juntas componen el 42,5\% de la reputación de una organización.

La recuperación de esa confianza perdida por parte de los ciudadanos es, pues, necesaria para que la empresa prospere tanto de forma económica como social en la actual «economía de los intangibles y de la reputación corporativa» (Alloza, Carreras y Carreras, 2013, p. 27), en la que una empresa será considerada como una buena empresa sólo si obtiene una buena reputación (Villafañe, 2013). Por este motivo, el carácter voluntario de la puesta en marcha de acciones en materia de RSC es esencial, y no deberán ser consideradas como tal aquellas medidas exigidas legalmente (Velasco, 2006, p. 21).

Por este motivo, muchas organizaciones se aprovechan de ello para realizar greenwashing, una práctica consistente en la difusión de informaciones inciertas acerca de la toma de medidas en materia medioambiental; unas informaciones que producen "una imagen distorsionada y tendenciosa a favor de los aspectos 'verdes', interpretados como positivos por los consumidores» (Hallama et al., 2011, pp. 1-2) y que se manifiesta, según Salas (2018, p. 34), mediante: 1) productos calificados como sostenibles que, en realidad, están compuestos por elementos artificiales y 2) eslóganes que, para llamar la atención de los consumidores, propagan una imagen ecologista incierta.

Pero las empresas se encuentran con un nuevo consumidor: un consumidor al que le gusta estar informado, que desconfía de los mensajes emitidos por las organizaciones y que busca en Internet opiniones sobre otros usuarios acerca de un producto antes de adquirirlo (Pérez y Clavijo, 2017, p. 233). Además, es un consumidor que, en alguna ocasión, su actitud acerca de la adquisición de un producto ha sido negativa hacia aquellas empresas no responsables (Pricewaterhousecoopers, 2004, p. 9), y que está dispuesto a pagar un precio mayor por productos sostenibles (González, 2015, p. 82).

A ese nuevo consumidor se le ha denominado como prosumer, en tanto a su experiencia en compras; como crossumer, como distribuidor y co-productor; como fansumer, establece conexiones con la marca y como ressumer, aquel que «exige a las empresas la contribución a la mejora de la sociedad [...] y una comunicación fluida y dialógica que le permita estar informado de las iniciativas y proyectos llevados a cabo por la empresa» (Ros y Castelló, 2011, pp. 49-50).

Además, los nuevos clientes consultan en Internet las opiniones que otros usuarios manifiestan sobre un producto (tanto su calidad como el proceso de envío y el postventa) antes de proceder con la compra del mismo (Martínez y De Garcillán, 2016, p. 89) y, además, los blogs de moda y, más reciente, la figura de los influencers, median en su decisión de compra (ibíd., p. 103), por lo que la comunicación entre empresa-usuario y viceversa «deja de ser unidireccional para beneficiarse de las opiniones y gustos que los usuarios vierten continuamente» (Vaño, Calduch-Losa y Orduña-Malea, 2016, p. 63). Ante ello, las empresas deben estar preparadas ante posibles comentarios negativos para saber y poder gestionarlos correctamente (IAB Spain, 2010, p. 18), pues un comentario negativo puede afectar a la reputación de la empresa (Villafañe, 2013, p. 3) y, de no ser bien gestionado, puede desencadenar en una crisis reputacional (Leiva-Aguilera, 2012, p. 72).

Es, por este motivo, por lo que la gestión de las comunicaciones (tanto de forma interna como de forma externa, online y offline) que han de ser, ante todo, bidireccionales, tiene una gran relevancia en las organizaciones actuales, ya que "todo lo que realiza una empresa comunica valores» (Benavides y Villagra, 2005, p. 151), y 
dichas comunicaciones definen «la comprensión social de los comportamientos de la empresa, convirtiendo a los públicos en instrumentos imprescindibles para el desarrollo y el reconocimiento reputacional de la organización» (ibíd., p. 152).

En concreto, la comunicación de la RSC, conlleva, sobre todo en el paradigma actual, "una importante carga comercial y en especial de diferenciación con competidores» (Velasco, 2006, p. 148), en el que "compartir, comunicar, conversar y cooperar son los cuatro pilares» (Ros y Castelló, 2011, p. 52). Y en el que, además, controlar lo que se dice sobre las organizaciones es una ardua tarea, por lo que la reputación de las mismas puede estar en peligro por un cualquier error cometido. Por esto, las empresas deben saber que su imagen dependerá de dos aspectos, como explican Fondevila, Del Olmo y Bravo (2012, p. 95): «lo que dice de sí misma [...] así como la imagen que sobre ella se crea su público y las personas que pueden opinar al respecto».

Realizar acciones de RSC y no comunicarlo a los públicos interesados, para ellos, significa no hacerlas. De poco sirve realizar acciones responsables cuando no son compartidas con los stakeholders, principales demandantes y afectados por las mismas. Por tanto, podemos afirmar que no hay RSC sin comunicación y que, si una organización quiere prosperar en un entorno tan competitivo como el actual, debe distinguirse de sus competidores siendo ética tanto en sus acciones como en sus comunicaciones.

\section{La sensibilización medioambiental en el sector de la moda}

Cada vez, la sociedad está tomando más conciencia del impacto ambiental que produce la moda rápida. Ya sea por su sensibilización sobre el estado del medio ambiente o bien por los diversos estudios publicados sobre ello, que llegan a los consumidores mediante su difusión en los medios de comunicación, así como publicaciones en revistas de moda como Vogue, que incluye, en muchas de sus portadas recientes, la palabra 'sostenibilidad', además de diversas entrevistas a diseñadores de moda que apuestan por un modelo de producción más lento y ecológico.

Tal es la gravedad de la situación, que la ONU ha tenido que solicitar a la industria textil, a la cual considera como "emergencia medioambiental» (Moda.es, 2018), un cambio de modelo enfocado hacia la sostenibilidad, poniendo como buen ejemplo de ello a la empresa sueca H\&M (Diarioresponsable.com, 2018). Pero la moda rápida no es sólo cuestionada por su impacto ambiental sino también el social, a raíz del derrumbe del edificio Rana Plaza en Bangladesh, en 2013, de ahí la creciente importancia de la gestión de la RSC, en todas sus dimensiones, en el sector textil (Martínez-Barreiro, 2016, p. 121), pues «una empresa será sostenibilista si es ambientalmente limpia, socialmente justa y económicamente viable» (Carrera-Gallisà, 2017, p. 24). Sin embargo, en palabras de WWF, este sector está muy lejos de ser sostenible en pos del medio ambiente debido al gran impacto que en el mismo genera en todas las fases del ciclo de vida de las prendas (2017, p. 8).

La industria textil, junto a la de calzado, generaron entre el 5 y el $10 \%$ de la polución a nivel mundial en el año 2016, siendo los procesos de tintado, la preparación del hilo y la producción de fibra los más contaminantes de la industria (Quantis y ClimateWorks Foundation, 2018, pp. 18-19). Pero en todos los procesos de producción de la moda rápida generan algún tipo de contaminación (Greenpeace, 2016): para la fabricación de materiales sintéticos, se extrae y se refina el petróleo, y muchos pesticidas y fertilizantes se utilizan en la recogida de materiales como el algodón (p. 3). 
El carbón proporciona energía a las centrales eléctricas en el proceso de confección de los productos, y esas fábricas textiles utilizan grandes cantidades de energía y productos químicos, que acaban contaminando los ríos de países asiáticos. Incluso hay un dicho popular que señala que «mirando el agua de los ríos de algunas ciudades de China se puede averiguar el color tendencia de las colecciones que lucen los occidentales» (Barrio, 2013).

Algunos de estos tóxicos son vertidos por parte de empresas proveedoras de grandes marcas del sector, como Benetton, Inditex, Adidas, Mango, H\&M o Nike, entre otros (Greenpeace, 2011, p. 6), y tienen una alta toxicidad tanto para las personas que visten las prendas como para los animales que habitan dichos ríos o mares (Greenpeace, 2012, p. 11). Y, además, los ciudadanos contribuimos a la intoxicación de las aguas, sin quererlo y sin saberlo, pues los materiales tóxicos con los que las prendas que vestimos son fabricadas se liberan al lavarlas (ídem).

El transporte hacia los centros de distribución también genera CO2, y las prendas que la gente ya no desea son finalmente incineradas o abandonadas en vertederos, y no recicladas, aunque las mismas empresas de moda no lo quieran reconocer (Dean, 2017 , p. 16). Esto se incrementa con las compras online, pues las mismas requieren un transporte desde los centros de distribución hacia los domicilios de los compradores. Y, del mismo modo, los desplazamientos de los consumidores hacia los comercios para adquirir productos también produce contaminación.

En el modelo económico de la moda rápida, los consumidores adquirimos más ropa que en épocas anteriores, las vestimos pocas veces o ni tan siquiera las estrenamos, y se tratan como productos desechables, sobre todo dada la baja calidad de algunas de las prendas que atiende a estándares calidad-precio (Greenpeace, 2016, p. 2). Mientras las marcas de lujo ofrecen dos temporadas al año (primavera/verano; otoño/invierno), las marcas de moda rápida ofrecen productos nuevos cada pocas semanas, con tendencias que coinciden con dichas dos temporadas o colecciones intermedias.

Asimismo, las colaboraciones entre marcas y artistas también conlleva al exceso de temporadas al año, que conllevan la fabricación de miles de prendas, siendo muchas de ellas no vendidas y rebajadas en periodos especiales (incluso entre temporadas, que incluso sirve como reclamo de consumo de nuevas temporadas) y, con ello, un mayor impacto ambiental. Esto es posible debido al acortamiento de los procesos de diseño y producción de las prendas, que se ha reducido de 6 meses a 3 y 4 semanas para la primera fase y de 3 meses a 7 días en la segunda de ellas, siendo pionera de ello la gallega Inditex, reduciéndose un $80 \%$ en conjunto (Martínez-Barreiro, 2008, p. 115), y también debido a que las prendas procedentes de la moda rápida se inspiran en los desfiles de alta costura y se copian, literalmente, dichos diseños, adaptándose a un ciudadano global abaratando tanto su precio como los costes y el tiempo de su producción (Martínez-Barreiro, 2016, pp. 115-116).

Como alternativa a la moda rápida, han surgido nuevas economías con la finalidad de «buscar respuesta a los graves desafíos sociales y medioambientales que el paradigma actual nos presenta» (OCU, 2019, p. 12), como el movimiento slow o moda lenta, también conocido como moda sostenible o moda ética, término utilizado por primera vez por Kate Fletcher (2007) para definir a una nueva economía de producción más lenta, que no se basa en el tiempo como la moda rápida, sino en la calidad y durabilidad de los productos, fabricados de forma más sostenible y considerando a los diseñadores locales; la economía circular o upcycling, consistente en la reutilización de los productos para la fabricación de nuevas prendas en lugar de ser desechados (Carrera-Gallisà, 2017, p. 32) o la compra-venta de productos de segunda mano (que 
incluso se ha puesto de moda), entre muchas otras (OCU, 2019, pp. 13-21), todas ellas enfoques diferentes en los que «diseñadores, compradores, distribuidores y consumidores son conscientes del impacto de los productos de vestir sobre las personas y los ecosistemas» (Martínez-Barreiro, 2016, p. 122).

Muchas empresas apuestan por estas nuevas economías, que nacen con la misión y la visión de ofrecer moda sostenible sin impacto ambiental y, aunque la mayoría de ellas son de reciente creación, algunas llevan varias décadas en el sector, como es el caso de la pionera People Tree, de origen británico. Y es que, además, suplen las demandas de un nuevo consumidor responsable, interesado en conocer qué hacen las marcas de moda para minimizar su huella ambiental, de dónde provienen los materiales con los que se fabrican las prendas, las acciones que llevan a cabo las organizaciones para proteger los derechos humanos de sus trabajadores o cómo fueron fabricados los productos ofertados (Fashion Revolution, 2018, pp. 9-11).

Además, muchos son los influencers o los famosos, que comparten en sus RRSS sus estilos de vida sostenibles, como Joaquin Phoenix, quien recicla su traje en cada entrega de premios del cine; un esmoquin realizado por Stella McCartney, una de las pioneras en el sector de la moda sostenible, aunque su marca es catalogada como marca de lujo, por lo que sus prendas no son asequibles para el público medio. O Emma Watson, icono de la moda sostenible, quien viste siempre con prendas eco-friendly, tanto en su día a día como en las presentaciones de películas o en la gira de presentación de las mismas, pues incluso creó una cuenta en Instagram para detallar, a sus seguidores, la procedencia de sus prendas, accesorios y maquillajes.

Marcas de alta costura también apuestan por alguna de las nuevas economías, como es el caso de María Escoté que recurre al upcycling en colaboración con Wallapop; o Prada, que en 2019 lanzó la colección Re-Nylon, una línea de bolsos realizada con materiales reutilizados. Del mismo modo, los grandes grupos del lujo también apuestan por la sostenibilidad: el grupo LVMH (en el que se enmarcan casas como Dior, Fendi, Louis Vuitton, Loewe, entre otras), mediante la política medioambiental LIFE (LVMH Initiatives For the Environment program), planteó cuatro objetivos en todas sus casas para 2020: reducir la huella ambiental de todos sus productos, implementar los mejores estándares en todas las cadenas de suministro, reducir las emisiones de CO2 y mejorar los indicadores de desempeño ambiental (LVMH, 2019), o el grupo Kering (Gucci, Saint Laurent, Balenciaga, Alexander McQueen, entre otras), cuya estrategia sostenible a alcanzar en 2025 se basa en tres pilares: Care (usando herramientas innovadoras y nuevas prácticas para reducir su impacto ambiental), Collaborate (promoción de la diversidad y la paridad y creación de políticas de empleo éticas) y Create (uso de materias primas innovadoras o fibras recicladas en la confección de sus prendas).

Por otro lado, grandes marcas de lujo como Versace, Prada o Gucci declararon su política anti-pieles para las próximas colecciones (Vogue, 2019). A pesar de ello, los productos de la última colección como los accesorios en dichas tres marcas continúan siendo fabricados con piel pero, aunque antes se especificaba el origen del cuero (bovino, caprino, equino...), ahora sólo se especifica piel, pero tampoco piel sintética. Un comportamiento que una parte de la sociedad considerará como maltrato animal y, por tanto, poco ético pero, por otra parte, los clientes usuales del lujo pueden considerar comprar productos que no estén fabricados con cuero animal alegando la calidad del mismo. Así que esto es un reto para dichas organizaciones: si o bien adaptarse a las demandas de los nuevos consumidores o seguir cumpliendo las preferencias de su público asiduo. 
El seguimiento de nuevas economías es posible en el sector del lujo debido a que sus procesos, tanto el de diseño como el de producción, son más lentos y más cuidados que los de la moda rápida. Pero, ¿qué hacen al respecto las empresas de moda rápida, que no nacieron bajo premisas de sostenibilidad, en lo referido a la reducción de su impacto ambiental? Y ¿cómo lo comunican a ese nuevo consumidor, más responsable y más preocupado por su entorno, y cómo se adaptan a sus nuevas demandas? ¿Existe alguna diferencia en las comunicaciones de RSC de empresas de moda rápida y moda lenta? Estos son algunos de los objetivos de la presente investigación, que serán explicados con más detalle a continuación.

\section{Objetivos}

El objetivo general de este estudio es analizar las comunicaciones sobre RSC en materia medioambiental en páginas web y en la red social Facebook en empresas de moda rápida y moda lenta y comprobar, si existe o no, una diferenciación entre las comunicaciones de ambos tipos de empresa.

Los objetivos específicos son:

- Conocer qué medidas medioambientales llevan a cabo tanto las empresas de moda rápida como las de moda lenta.

- Comprobar si la información sobre responsabilidad medioambiental en páginas web es compartida en redes sociales y en qué medida.

- Conocer en qué medida y con qué frecuencia dichas empresas comunican sus responsabilidades medioambientales en la red social Facebook.

- Conocer qué tipo de comunicaciones en redes sociales suelen llevar a cabo ambos tipos de empresa.

- Averiguar la frecuencia de uso de Facebook por ambos tipos de empresa.

\section{Metodología utilizada}

Para la consecución de dichos objetivos, este trabajo utiliza la técnica de análisis de contenido cualitativo, por lo que no parte de una o varias hipótesis, sino que las mismas «se van generando durante el proceso, se afinan conforme se recaban más datos, se modifican según los resultados y no se prueban estadísticamente» (Sampieri, Fernández y Baptista, 2014, p. 357). Dicha técnica se centra en observar las comunicaciones en RSC de empresas de moda rápida y moda lenta en la red social Facebook, escogida por ser la segunda más utilizada por los usuarios por detrás de Whatsapp (IAB Spain, 2019, p. 18), durante el mes de enero de 2020. Asimismo, se realizará una observación de las acciones sostenibles comunicadas en sus páginas web, así como e-commerce de cada una de sus marcas. De este modo, podremos comparar la comunicación entre web y Facebook, así como conocer qué acciones realizan y si se comparten con sus consumidores.

La muestra del estudio se obtuvo mediante un muestreo no probabilístico, o muestreo de conveniencia, en concreto el muestreo intencional o deliberado, en el cual, «el investigador decide según los objetivos, [...] considerando aquellas unidades supuestamente típicas de la población que se desea conocer» (Canales, Alvarado y Pineda, 1994, p. 119), y es homogénea debido a que las unidades escogidas "poseen un mismo 
perfil o características, o bien comparten rasgos similares» (Sampieri, 2019, p. 388). La misma puede dividirse en dos grupos: un primer grupo, en el que se engloban los cinco grandes grupos de moda rápida: Inditex, C\&A, H\&M, Mango y Benetton, y un segundo grupo formado por cinco empresas de moda lenta: Finisterre, People Tree, Outland Denim, SKFK y MUD Jeans.

Para la selección de las organizaciones que forman parte del segundo de los grupos, se utilizó la aplicación Good On You, que califica a las empresas como éticas o no en una escala del 1 (evitamos) al 5 (genial), según variables relacionadas con la gente, en cuestiones de derechos laborales; el planeta, en cuanto a la reducción de su impacto ambiental y los animales, el uso de sus pieles o pelajes, como el caso de la lana, basándose en el Ethical Fashion Report del año 2018 y su propia investigación. Así pues, las empresas seleccionadas de este grupo tienen una cierta homogeneidad con la muestra de empresas de moda rápida, tanto por su ejercicio como el rango de precios pero, por el contrario, son catalogadas como éticas con calificaciones de 4-5 por Good On You.

Dicha categorización positiva a empresas de moda lenta, a diferencia de las de moda rápida, que todas han sido calificadas como no éticas por diversos, nos hará observar unas comunicaciones entre empresas calificadas como sostenibles o no lo suficientemente sostenibles por una organización imparcial que se basa en estudios e investigaciones propias para medir sus calificaciones.

\section{Resultados}

En primer lugar, en lo referido a los seguidores en su perfil de Facebook, se observa que, mientras que las marcas de moda rápida tienen millones de seguidores, siendo H\&M (37M) la que más seguidores tiene, seguido de Zara (27) y Pull\&Bear (11), y en último lugar, M. Dutti (4) y Uterqüe (3). Las marcas de moda lenta miden sus seguidores en miles, siendo SKFK (205K) la que mayores seguidores presenta, seguida de People Tree (74K) y, en último lugar, Outland Denim (8).

En lo referido a su presencia en los distintos perfiles de RRSS, Facebook e Instagram son las más utilizadas por la totalidad de la muestra, seguidas de Twitter y Pinterest (Gráfico 1):

Gráfico 1. Perfiles en redes sociales
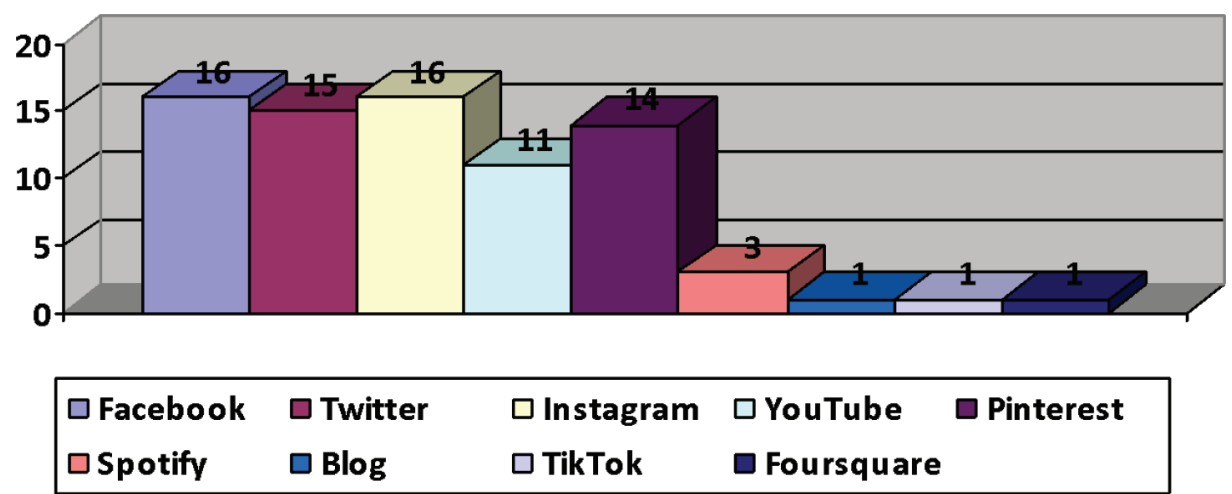

Fuente: Elaboración propia 
En cuanto al número de publicaciones, se observa una diferencia entre las marcas de moda rápida y moda lenta: mientras que las primeras publican una media de 17 post al mes, siendo Pull\&Bear (54), Massimo Dutti y Bershka (34) las que más publican, a diferencia de Zara (14), Mango (9) y Benetton (3), las segundas publican una media de 13 post/mes, siendo People Tree (34) y Finisterre (11) las que más comunican por este medio y Outland Denim (9) y MUD Jeans (3) las que menos.

Sólo H \& M, como empresa de moda rápida, comunica su sostenibilidad una vez, en concreto un producto de su colección Conscious. Las publicaciones más comunes entre las mismas son (Gráfico 2):

Gráfico 2. Tipo de publicaciones en moda rápida

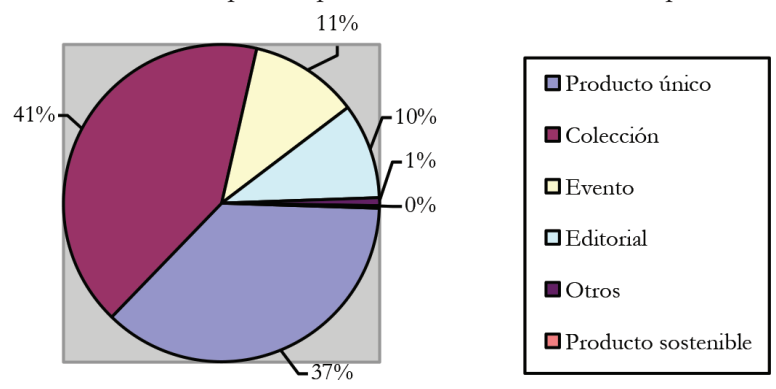

Fuente: Elaboración propia

Esto es interesante puesto que Inditex tiene la colección sostenible Join Life (presente en Zara, Pull\&Bear, Bershka, Oysho y Massimo Dutti, pero no en Uterqüe y Stradivarius), y H\&M la colección Conscious, ambas realizadas con materiales sostenibles o producidas de forma sostenible, aunque esto varía en cada producto y la información se detalla en los e-commerce de cada organización. Pero no todos los productos de las colecciones presentan esta información, sino que gran parte de los productos sigue sin fabricarse de manera sostenible y sin materiales ecológicos.

Es importante destacar que, en aquellas promociones de un determinado producto de Inditex, se publican, hasta en diez ocasiones, productos que pertenecen a la colección Join Life y esto no se comunica en Facebook. Ocurre en el caso de Zara y Pull \& Bear. Por otro lado, Zara ha publicado, en el mes de febrero de 2020, post en los que presenta su colección realizada con algodón ecológico.

Los tipos de publicaciones más utilizados por las empresas de moda lenta son (Gráfico 3):

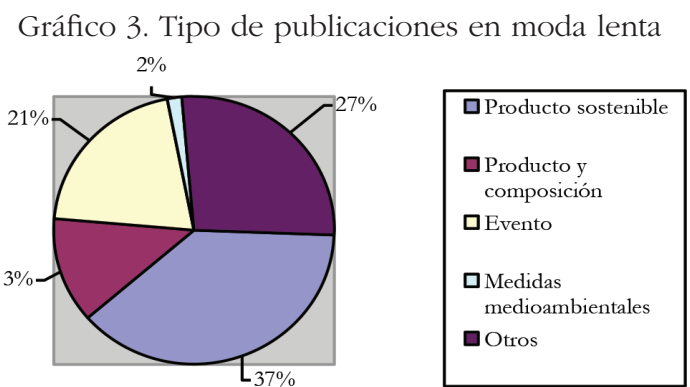

Fuente: Elaboración propia 
Este tipo de empresas comunican la sostenibilidad tanto en el proceso de fabricación como en los materiales de los productos, en menor medida. También suelen publicar mucho acerca de acciones de RSC, como donaciones para los incendios de Australia, participaciones e invitaciones a los seguidores a eventos como charlas sobre los microplásticos y sus devastadores efectos en el mar o su participación en pasarelas de moda lenta.

El número de publicaciones en Facebook al mes es mayor en empresas de moda rápida, siendo Pull\&Bear (54) la que más, seguida de Bershka y Massimo Dutti (34), aunque People Tree, de moda lenta, también genera esa cantidad, situándose incluso por delante de Mango y Benetton (9 y 3, respectivamente), quienes también son superadas por H\&M (30), Stradivarius (20) o C\&A (18), en moda rápida, y Finisterre (11) y SKFK (10) en moda lenta, por lo que no hay una homogeneidad entre empresas de la misma producción.

En cuanto a los contenidos de las publicaciones, las imágenes y los vídeos son los recursos más utilizados por ambos tipos de empresa (la totalidad en moda rápida y un $82 \%$ en moda lenta), seguido de URL, que suele redirigir o bien al producto anunciado o a diferentes categorías (como novedades o una colección en concreto), los más utilizados. A estos les preceden los emojis (60,7\%) y los hashtags (36,8\%) en moda rápida, y el caso contrario en moda lenta ( $17,9 \%$ y $76 \%$, respectivamente).

En lo referido a los mensajes de los post, se observa lo siguiente (Gráfico 4):

Gráfico 4. Tipo de comunicaciones en post
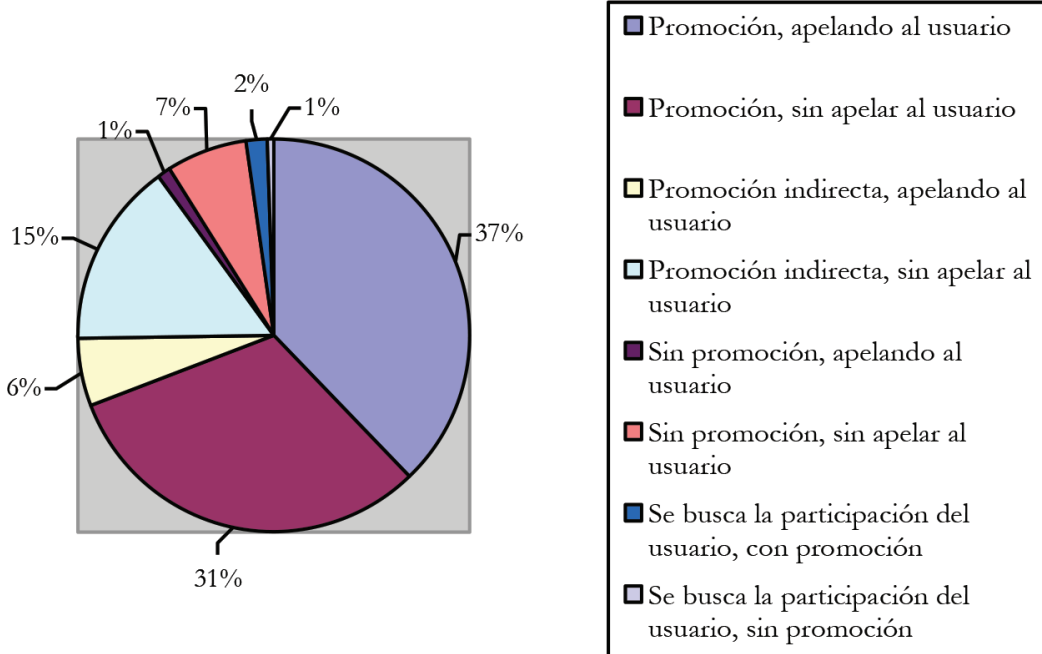

$\square$ Sin promoción, sin apelar al usuario

$\square$ Se busca la participación del usuario, con promoción

$\square$ Se busca la participación del usuario, sin promoción

Fuente: Elaboración propia

Se aprecia una semejanza entre ambos tipos de empresa: mientras que las comunicaciones más utilizadas son: 1) la promoción apelando al usuario por parte de todas las empresas de moda rápida y un 80\% las de moda lenta; 2) la promoción sin apelar al usuario, por parte del primer grupo es utilizada por un 63,6\% del total y, en el segundo, un $80 \%$ y en el tercer lugar se aprecia una diferencia: mientras que este puesto en moda rápida lo ocupan las publicaciones indirectas sin apelar al usuario, aunque con un porcentaje muy menor a los anteriores (5.4\%), en moda lenta lo 
ocupan las publicaciones sin promoción y sin apelar al usuario. Las menos utilizadas son, en moda rápida, no se realizan publicaciones en las que se busca la participación del usuario, sin promoción y, en moda lenta, no hacen promociones indirectas apelando al usuario ni sin promoción apelando al usuario.

El lenguaje utilizado, en la totalidad de ambos tipos de empresa, no pretende conectar con el público, sino que se utiliza un lenguaje que hace referencia a lo que se anuncia de forma general. En cuanto al lenguaje que sí pretende conectar con los seguidores, es utilizado en alguna ocasión por un $45.4 \%$ en moda rápida y un $60 \%$ en moda lenta, en las que se hace referencia a los mismos y se los relaciona con lo comunicado para, así, establecer una conexión entre ambos.

En lo referido al engagement de los usuarios, la reacción preferida por los mismos es el me gusta, presente en todas las publicaciones de ambas empresas, seguido de me encanta, con totalidad en los post en moda rápida y en un $80 \%$ en moda lenta. Las reacciones menos presentes han sido me entristece en moda rápida (45.4\%) y me divierte no se observa en el caso de moda lenta. Las publicaciones han sido compartidas con los fans en la totalidad de los post de ambas organizaciones.

Todas las marcas de moda rápida reciben comentarios en sus publicaciones, excepto un caso de moda lenta: un 82,3\% en las publicaciones en moda rápida y un 32.8\% en moda lenta. Los tipos de comentarios que más se observan en las publicaciones por parte de los fans son las reacciones positivas a lo publicado, presente en la totalidad de publicaciones de moda rápida y el 60\% en moda lenta. El segundo tipo de comentario más común en moda rápida son quejas, presentes en los comentarios de todas las organizaciones de moda rápida excepto Benetton; y, en moda lenta, otros, que tienen lugar en el 60\% de los casos, y son todos ellos comentarios en los que usuarios etiquetan a otros usuarios. En tercer lugar, se encuentran las preguntas a la marca sobre la misma o algún producto, que tiene lugar en todas las publicaciones de moda rápida y en el $80 \%$ en moda lenta.

\section{Discusión de resultados}

A partir de los resultados mostrados, y relacionándolos con los objetivos propuestos, se concluye que, en primer lugar, las empresas de moda rápida realizan acciones medioambientales y se comunican mediante la publicación de memorias de sostenibilidad, en todos los casos, al igual que un código ético. Inditex, H \& M y C \& A tienen colecciones producidas y confeccionadas de forma sostenible, y se ofrecen junto a otros productos que no, pues dicha información sostenible se anuncia en las prendas que pertenecen a dicha colección pero no aparece en el resto de prendas. Por tanto, afirmamos que su colección no es 100\% sostenible, pero cada una de ellas ha fijado unos plazos para conseguirlo.

En el caso de las empresas de moda lenta, se observa que sólo MUD Jeans elabora informes de sostenibilidad, y todas excepto People Tree elaboran un código de conducta. A diferencia que las de moda rápida, los productos que ofrecen estas empresas son $100 \%$ sostenibles en su conjunto, sin destacar una única colección que lo es. Esto demuestra su compromiso medioambiental (y social), y la diferencia que radica entre las de moda rápida, cuyos objetivos iniciales en el momento de su fundación se circunscriben únicamente a objetivos económicos y no de responsabilidad con su entorno, unos valores, misión y visión con las que sí fueron fundadas las empresas de moda lenta analizadas. 
Todas ellas participan con ONG como Better Cotton Initiative, que promueve, en el sector textil, una producción de algodón sostenible tanto para las personas implicadas en el proceso de producción como al planeta y al sector; la coalición Free Fur Alliance, de la que todas excepto Benetton forman parte, y que fomenta el trato ético con los animales para el uso de sus pieles, como la crianza de los mismos en granjas especializadas y sin ningún tipo de maltrato, aunque dicha ética puede ser desmontada al utilizar dichas pieles para la confección de prendas.

Inditex y C\&A forman parte de la iniciativa CEO Water Mandate, del Pacto Mundial de las Naciones Unidas, que impulsa una administración sostenible del agua, a diferencia de H\&M, Mango y Benetton, y todas excepto Mango colaboran con el programa Zero Discharge of Hazardous Chemicals (ZDHC), cuya misión es reducir el impacto medioambiental que generan las empresas, tanto proveedores y fabricantes de productos químicos y otras organizaciones. Por su parte, las empresas de moda lenta no colaboran con ninguna de estas acciones, sólo MUD Jeans con la iniciativa Fur Free Alliance. Sin embargo, se observa que People Tree trabaja en conjunto con asociaciones de Fair Trade (Comercio Justo) o sigue estándares como los Global Organization Textile Standards.

Por otro lado, Outland Denim recibe una calificación de A+ por el Rating Ethical Fashion Report, en el cual no aparecen el resto de empresas de moda lenta, pero sí algunas de moda rápida como Inditex, que obtiene un A, H\&M un B+ y Mango una C. En cuanto a sus calificaciones en Good on You, destaca C \& A con una evaluación de 4/5, seguida de Inditex y H \& M, que obtienen un 3/5, y Mango y Benetton, un 2/5. Por otra parte, las empresas de moda lenta obtienen todas una calificación excelente de 5/5 excepto Finisterre, que obtiene un 4/5, al igual que C \& A.

A pesar de ello, y de otras medidas medioambientales que dichas empresas realizan y cuyo más profundo análisis se pretende desarrollar en futuras investigaciones, ninguna de las anteriormente citadas se promociona en Facebook al menos, durante el periodo analizado, sólo H \& M promueve una vez una prenda de su colección Conscious. Esto bien puede deberse a que las mismas están todavía estudiando si a su target le interesa todo lo relacionado con la sostenibilidad de las prendas que adquiere o bien a que la producción sostenible de la totalidad de la producción todavía no es posible, aunque todas ellas lo plantean.

Por otro lado, las empresas de moda lenta sí comunican sus acciones medioambientales en Facebook, tanto la sostenibilidad del negocio en su conjunto y su compromiso medioambiental. A diferencia del caso de la moda rápida, este tipo de organizaciones sabe que a sus seguidores les interesa adquirir ropa totalmente sostenible y, además, fueron fundadas bajo el paraguas de la sostenibilidad, por lo que sus comunicaciones en Facebook coinciden con su visión del negocio, lejos del greenwashing.

Se observa que no hay homogeneidad en el uso de Facebook por las empresas con el mismo ritmo de producción, sino que hay organizaciones de moda lenta que publican más que las de moda rápida, al igual que el tipo de publicaciones, que en su mayoría es la promoción de producto en ambos casos. En contraposición, sí existe una diferencia en el uso de distintos perfiles de RRSS, pues las empresas de moda rápida suelen estar más presentes que las de moda lenta, aunque en la mayoría de ellas (las más utilizadas por los usuarios, como Facebook, Twitter, Instagram o Pinterest), ambas coinciden.

Asimismo, se pueden establecer una serie de correlaciones que no podemos detallar en profundidad debido a las características demandadas para esta investigación, pero su estudio puede desembocar en futuras investigaciones. Del mismo modo, 
también se plantea analizar la totalidad de productos que las empresas de moda rápida ofrecen como sostenibles, y compararlo con aquellos productos que se ofrecen en los que esto no se comunica para, así, saber qué diferencia de la producción total significa cada uno de ellos. También se plantea el análisis de las comunicaciones de RSC en el sector de la moda de lujo o sobre la información sobre medidas de RSC que el consumidor tiene sobre el sector textil.

\section{Conclusiones}

La falta de comunicación sobre RSC en Facebook en el caso de empresas de moda rápida es una práctica contraria a la comunicación sobre la misma publicada en su página web. El motivo exacto de ello se desconoce, pero podemos deducir que su estrategia medioambiental, según sus planificaciones, no será totalmente efectiva en la totalidad de su producción hasta dentro de un periodo máximo de cinco años, según las mismas empresas comunican. Al contrario, en moda lenta es una práctica habitual y efectiva en su totalidad debido a que la sostenibilidad es una práctica inherente en la misión, visión y valores de la organización.

Por tanto, al no realizar dichas comunicaciones de RSC en medios sociales por parte de las empresas de moda rápida, cabría preguntarnos si, realmente, están comunicándose al consumidor que, como stakebolder, es el principal beneficiario y demandante de las mismas. Porque sí es cierto que hay más información en los portales corporativos, pero la medida en la que los usuarios visitan dichos sitios, distintos a los e-commerce, es mucho menor que a los perfiles de RRSS.

Asimismo, sería interesante realizar un seguimiento de la evolución de las prácticas sostenibles por parte de las empresas de moda rápida durante los próximos años, tanto en su puesta en marcha como en la comunicación de las mismas, pues son en los que se proponen producir de manera 100\% sostenible. Y, además, porque no sólo puede efectuarse un cambio en las empresas, sino también por parte del consumidor, cuya responsabilidad puede expandirse a una cuota mayor de la ciudadanía.

Por otro lado, las bajas calificaciones que obtienen por parte de Good On You solo hace que evidenciar que las políticas de sostenibilidad en moda rápida no son suficientes y que las mismas deben mejorar para convertirse, en su totalidad, en empresas sostenibles, siendo la voluntariedad de las mismas un aspecto clave para ello, y no basar sus políticas meramente en obligaciones legales llevadas a cabo por las administraciones, tanto a nivel nacional como a nivel europeo.

El modelo de producción y confección sostenibles de las organizaciones de moda lenta analizadas se convierte en un ejemplo a seguir para las de moda rápida, aunque la economía en la que se rigen ambos tipos de empresa es totalmente diferente. Por este motivo, y como hace WWF, habría que preguntarse si la moda rápida, por su modelo de negocio consistente en una constante producción de periodicidad corta, podrá o no llegar a ser cien por cien sostenible en un futuro.

\section{Bibliografía}

Alloza, A., Carreras, E. y Carreras, A. (2013). Reputación Corporativa. Madrid: Lid Editorial Andreu, A. y Fernández, J.L. (2011). De la RSC a la sostenibilidad corporativa: una evolución necesaria para la creación de valor. Harvard Deusto Business Review 
Barrio, E. (2013). Moda y RSC, tejiendo compromisos. En Compromisoempresarial.com. Recuperado de https://www.compromisoempresarial.com/rsc/2013/04/moda-y-rsc-tejiendo-compromisos/

Barrio Fraile, E. (2019). Responsabilidad Social Corporativa. De la noción a la gestión. Barcelona: Editorial UOC

Benavides Delgado, J. y Villagra García, N. (2005). Breves reflexiones sobre la comunicación de la Responsabilidad Social Corporativa: un reto para las empresas del s. XXI. En N. García Villagra (Ed.). La comunicación de la Responsabilidad Social Corporativa (pp. 143-164). Cátedra Javier Benjumea de Ética Económica y Empresarial. Memorias Académicas, 2. Madrid: Universidad Pontificia Comillas

Better Cotton Initiative. About BCI. Recuperado de https://bettercotton.org/about-bci/

Bowen, H. R. (1953). Social responsibility of the businessman. New York: Harper \& Row

Canales, F. H., de Alvarado, E. L. y Pineda, E.B. (1994) [1986]. Metodología de la investigación. Manual para el desarrollo de personal de salud. Washington: Organización Panamericana de la Salud.

Carrera-Gallisà, Enric (2017). Los retos sostenibilistas del sector textil. En Revista de Química e Industria Textil, (220), pp. 20-32 Recuperado de http://hdl.handle.net/2117/103614

Castelló Martínez, A. (2010a). La orientación empresarial hacia el cliente en la Web 2.0. En Miguel Hernández Communication Journal, (1), pp. 99-131. ISSN: 1989-8681

Castelló Martínez, A. (2010b). La sensibilización medioambiental en redes sociales online. En Fisec-Estrategias, (13), pp. 23-46. Recuperado de http://www.cienciared.com.ar/ra/doc. php?n=1261

Centro de Información de las Naciones Unidas para España (CINU). El acuerdo mundial. Obteniendo el Compromiso de las Corporaciones y la Sociedad Civil para Enfrentar los Desafíos de la Globalización. Recuperado de https://www.un.org/spanish/milenio/np4milenio.htm

CEO Water Mandate, UN Global Compact. What is the mandate? Recuperado de https://ceowatermandate.org/about/what-is-the-mandate/

Comisión Europea (2001). Libro Verde. Fomentar un marco europeo para la responsabilidad social de las empresas. Recuperado de https://www.europarl.europa.eu/meetdocs/ committees/deve/20020122/com(2001)366_es.pdf

Cortina, Adela (2005). Ética de la empresa, no sólo responsabilidad social. El País.es (20/08/2005) Recuperado de https://elpais.com/diario/2005/08/20/opinion/1124488806_850215.html

Crisófol, Francisco Javier, Segarra-Saavedra, Jesús y Cristófol-Rodríguez, Carmen (2019). La comunicación corporativa en Facebook de las marcas españolas de moda Zara y Mango. En Prisma Social, revista de ciencias sociales, (24), pp. 234-254. Recuperado de https:// revistaprismasocial.es/article/view/2808

Dean, Christina (2017). 'What happens to clothing that goes unsold? En Fashion Revolution, Loved Clothes Last. Recuperado de https://www.fashionrevolution.org/resources/fanzine2/

Diarioresponsable.com (2018). La ONU pide a la industria de la moda un cambio de modelo hacia la sostenibilidad. Recuperado de https://diarioresponsable.com/noticias/26769-laonu-pide-a-la-industria-de-la-moda-un-cambio-de-modelo-hacia-la-sostenibilidad

Escoté, M. [maria_escote] (29 de julio de 2019). Upcycling is coming for SS20 \#wallapopxmaríaescoté \#mariaescote [Imagen de Instagram] Recuperado de https://www.instagram. $\mathrm{com} / \mathrm{p} / \mathrm{B} 0 \mathrm{fhsi6HqJB} /$

Fashion Revolution (2018). Consumer survey report. Recuperado de https://www.fashionrevolution. org/resources/consumer-survey/

Fernández Fernández, J.L. (2005). De la "tesis" de Milton Friedman y el contrapunto de Edward Freeman, hacia un nuevo paradigma de empresa. En N. García Villagra (Ed.), La Comunicación de la Responsabilidad Social Corporativa (pp. 19-32). Cátedra Javier Benjumea de Ética Económica y Empresarial. Memorias Académicas, 2. Madrid: Universidad Pontificia Comillas

Fernández Izquierdo, M.A. y Muñoz Torres, M.J. (2008). El gobierno corporativo. En Fernández Izquierdo, M.A (et al.) (2008). El gobierno corporativo como motor de la responsabilidad 
social corporativa. Grupo de Investigación SoGRes - Castelló de la Plana: Publicacions de la Universitat Jaume I

Fletcher, Kate (2007). Slow fashion. En The Ecologist. Recuperado de: https://theecologist. org/2007/jun/01/slow-fashion

Fondevila, Joan Francesc, Del Olmo, Josep Lluis y Bravo, Vanessa (2012). Presencia y reputación digital en social media: comparativa en el sector de la moda. Fonseca, Journal of Communication, 5(5), pp. 90-113. Recuperado de https://revistas.usal.es/index.php/2172-9077/ article/view/12080

Fundación BBVA (septiembre 2019). Estudio Internacional de Valores Fundación BBVA. Primera parte: Valores y actitudes en Europa acerca de la esfera pública. Recuperado de https:// www.fbbva.es/wp-content/uploads/2019/09/Presentacion_Estudio_Valores_2019.pdf

Fur Free Alliance. Our Issues. Recuperado de https://www.furfreealliance.com/our-issues/

Good On You. How We Rate. Recuperado de https://goodonyou.eco/how-we-rate/

Gómez Nieto, B. y Martínez Domínguez, R. (2015). La comunicación online de la RSC. El caso de las empresas líderes del mercado minorista español. En Razón y Palabra, (92), pp. 1-25. Recuperado de https://www.redalyc.org/articulo.oa?id=1995/199543036067

González, J. (2015). La sostenibilidad ecológica en el desarrollo de productos textiles: Una Revisión de Literatura. En Realidad y Reflexión, (38), pp. 65-97. doi: 10.5377/ryr.v38i0.1833

González Esteban, E. (2007). La teoría de los stakeholders. Un puente para el desarrollo práctico de la ética empresarial y de la responsabilidad social corporativa. En Veritas. Revista de Filosofía y Teología, 2(17), pp. 205-226. Recuperado de https://www.redalyc.org/ pdf/2911/291122924002.pdf

Greenpeace (2011). Trapos Sucios. Contaminación tóxica del agua en China por marcas textiles internacionales. Recuperadodehttp://archivo-es.greenpeace.org/espana/es/reports/TraposSucios/

Greenpeace (2012). Puntadas tóxicas: El oscuro secreto de la moda. Cómo las grandes marcas hacen que los consumidores seamos cómplices del ciclo tóxico del agua. Recuperado de http://archivo-es.greenpeace.org/espana/es/reports/Puntadas-toxicas/

Greenpeace (2016). Timeout for fast fashion. Recuperado de https://www.greenpeace.org/archive-international/Global/international/briefings/toxics/2016/Fact-Sheet-Timeout-for-fast-fashion.pdf

Hallama, M.; Montlló Ribo, M.; Rofas Tudela, S. y Ciutat Vendrell, Genís (2011). El fenómeno del greenwashing y su impacto sobre los consumidores. Propuesta metodológica para su evaluación. En Aposta. Revista de Ciencias Sociales, (50), julio-septiembre, 2011, pp. 1-38.

IAB Spain (2010). Libro blanco de la comunicación en medios sociales. Recuperado de http:// www.iabspain.net/ver.php?mod=descargas

IAB Spain (2019). Estudio anual de redes sociales 2019. Recuperado de https://iabspain.es/ estudio/estudio-anual-de-redes-sociales-2019/

Iborra-Juan, M. y Peris-Suay, A. (2010). Reconstruyendo la confianza en las empresas mediante la responsabilidad social corporativa: una ilustración en las cadenas de suministros del sector textil. En Revista Globalización, Competitividad y Gobernabilidad, 4(1), pp. 102-118. doi: 10.3232/GCG.2010.V4.N1.07

Ipsos Global Advisor (2019). What worries the world - May 2019. Recuperado de https://www. ipsos.com/es-es/que-preocupa-al-mundo-mayo-2019

Jáuregui, R. (2012). La RSE en Europa y en España: La empresa en el siglo XXI. En J.I. Galán y A. Sáenz de Miera (Eds.). Reflexiones sobre la Responsabilidad Social Corporativa en el siglo XXI, pp. 19-46. Salamanca: Ediciones Universidad de Salamanca

Kering. Sustainability. Our Strategy. Recuperado de https://www.kering.com/en/sustainability/ our-strategy/

Kliksberg, Bernardo (2012). 'La crisis y la Responsabilidad Social Empresarial'. En Galán, José Ignacio y Sáenz de Miera, Antonio (eds.) (2012). Reflexiones sobre la Responsabilidad Social Corporativa en el siglo XXI, pp. 47-68. Salamanca: Ediciones Universidad de Salamanca 
LVMH (25 septiembre 2019). LIFE 2020: four environmental excellence objectives at LVMH. Recuperado de https://www.lvmh.com/news-documents/news/life-2020-four-environmentalexcellence-objectives-at-lvmh/

Martínez-Barreiro, A. (2008). Hacia un nuevo sistema de moda. El modelo Zara. En Revista Internacional de Sociología, (51), pp. 105-122. doi: 10.3989/ris.2008.i51.111

Martínez-Barreiro, A. (2016). Moda Sostenible. En Almarcha, A. (Coord.) Donde la sociología te lleve: miscelánea en torno a la figura de Benjamín González Rodríguez. Unisersidade da Coruña, Servizo de Publicacións

Martínez-Navarro, G. y De Garcillán, M. (2016). La influencia de los blogs de moda en el comportamiento del consumidor: un enfoque exploratorio. En Vivat Academia, (135), pp. 85-109. doi: 10.15178/va.2016.135.85-109

Merco (2019). El proceso de elaboración de Merco España 2019. Recuperado de http://www. merco.info/es/ranking-merco-empresas

Moda.es (2018). La ONU califica la industria del fast fashion de 'emergencia medioambiental'. Recuperado de https://www.modaes.es/back-stage/la-onu-califica-la-industria-del-fast-fashion-como-emergencia-medioambiental.html

Naciones Unidas (2015). La Asamblea General adopta la Agenda 2030 para el Desarrollo Sostenible. Recuperado de https://www.un.org/sustainabledevelopment/es/2015/09/la-asambleageneral-adopta-la-agenda-2030-para-el-desarrollo-sostenible/

OCU (2019). Otro consumo para un futuro mejor. Nuevas economías al servicio de las personas y el planeta. Recuperado de https://www.ocu.org/consumo-familia/consumo-colaborativo/ informe/moda-sostenible\#

Pérez Curiel, C. y Clavijo Ferreira, L. (2017). Comunicación y Social Media en las Empresas de Moda. Asos como caso de estudio. En Revista Prisma Social, (18), pp. 226-258. Recuperado de https://revistaprismasocial.es/article/view/1440

Prada. Prada Re-Nylon. Recuperado de https://www.prada.com/us/en/pradasphere/special-projects/prada-re-nylon-1.html

Pricewaterhousecoopers (2004). La actitud del consumidor hacia la Responsabilidad Social Corporativa (RSC). Recuperado de https://jussemper.org/Newsletters/Resources/Actitud_ Consum_Esp_RSC.pdf

Quantis y ClimateWorks Foundation (2018). Measuring Fashion: Insights from the Environmental Impact of the Global apparel and Footwear Industries study. Recuperado de: https:// quantis-intl.com/wp-content/uploads/2018/03/measuringfashion_globalimpactstudy_ full-report_quantis_cwf_2018a.pdf

Reputation Institute (2019). RepTrak España 2019. Recuperado de https://insights.reputationinstitute.com/reptrak-reports/spain-reptrak-2019

Ros-Diego, V. y Castelló Martínez, A. (2011). La comunicación de la responsabilidad en los medios sociales. En Revista Latina de Comunicación Social, (67), pp. 47-67. doi: 10.4185/ RLCS-067-947-047-067

Salas, H. (2018). El greenwashing y su repercusión en la ética empresarial. En Neumann Business Review, 4(1), pp. 28-43. doi: 10.22451/3002.nbr2018.vol4.1.10018

Sampieri, R., Fernández, C. y Baptista, P. (2014) [1991]. Metodología de la investigación. México: McGraw Hill

TETRA PAK (2019). The TETRA PAK Index 2019. Recuperado de https://www.ipsos.com/sites/ default/files/ct/news/documents/2019-11/tetra-pak-index-2019.pdf

Túñez López, J.M y Valarezo González, K. (2012). RSC: Reputación, Sensibilidad, Compromiso. En Chasqui, Revista Latinoamericana de Comunicación, (117), pp. 61-66. doi: 10.16921/ chasqui.v0i117.221

Velasco Osma, J.R. (2006). Fundamentos de la Responsabilidad Social Corporativa y su aplicación ambiental. Madrid: Dykinson

Vaño Planells, Maria, Calduch-Losa, Ángeles y Orduña-Malea, Enrique (2016). «Análisis descriptivo de la presencia web de las empresas españolas del sector de la moda." pp. 61-74 en 
Actas del Co-munica2: Congreso Internacional sobre redes sociales, editado por Universidad Politécnica de Valencia. Gandía: UPV. Recuperado de http://hdl.handle.net/10760/30957

Villafañe, Justo (2005). La gestión de los intangibles empresariales. En Comunicaçao e Sociedade, 8, pp. 101-113. doi: 10.17231/comsoc.8(2005).1185

Villafañe, Justo (2013). La buena empresa. Propuesta para una teoría de la reputación corporativa. Madrid: Pearson

Rámila, L. (2019) (22 de mayo de 2019). El Grupo Prada se une a la ola de renuncia a las pieles del mundo de la moda. Vogue.es. Recuperado de https://www.vogue.es/moda/news/ articulos/grupo-prada-prohibe-uso-pieles/40572

Roadmap to Zero. About us. Recuperado de https://www.roadmaptozero.com/about

WWF (2017). Changing fashion. The clothing and textile industry at the brink of radical transformation. Environmental rating and innovation report 2017. Recuperado de https://www.wwf. ch/sites/default/files/doc-2017-09/2017-09-WWF-Report-Changing_fashion_2017_EN.pdf 